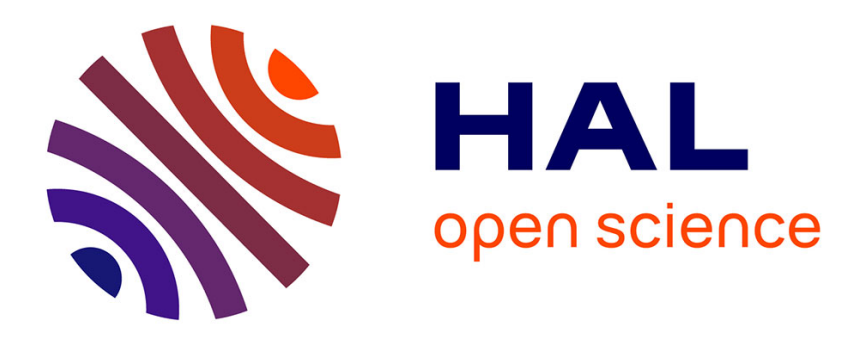

\title{
Capital social et Nuclei d'entreprises en Algérie
}

Cécile Perret

\section{To cite this version:}

Cécile Perret. Capital social et Nuclei d'entreprises en Algérie. Mondes en Développement, 2010, 1 (149), pp.105-116. 10.3917/med.149.0105 . hal-00951176

\section{HAL Id: hal-00951176 https://hal.science/hal-00951176}

Submitted on 25 Feb 2014

HAL is a multi-disciplinary open access archive for the deposit and dissemination of scientific research documents, whether they are published or not. The documents may come from teaching and research institutions in France or abroad, or from public or private research centers.
L'archive ouverte pluridisciplinaire HAL, est destinée au dépôt et à la diffusion de documents scientifiques de niveau recherche, publiés ou non, émanant des établissements d'enseignement et de recherche français ou étrangers, des laboratoires publics ou privés. 


\title{
Capital social et Nuclei d'entreprises en Algérie
}

\author{
Cécile PERRET ${ }^{1}$
}

$\mathrm{D}$

ans nombre de cas, la littérature se réfère à une conception humaniste et sociale du développement local qui est considéré comme "une démarche collective, un éveil des capacités de chacun, une expression de démocratisation réelle" (Denieuil 1999, 2). Aujourd'hui, le développement local combine une approche territoriale (initiative des acteurs locaux) avec le mouvement naissant des conséquences de la mondialisation, de la déconcentration et de la territorialisation par les États de leurs politiques publiques. Plus que jamais, l'enjeu est de savoir quel rôle la société civile doit jouer par le biais de la création locale d'entreprises, des initiatives sociales et associatives, pour faire face à l'exclusion.

Le paradigme du développement local se veut englobant, il vise à intégrer les multiples dimensions du développement: économique, sociale, culturelle, politique, etc. Il souligne l'importance des relations sociales comprises "comme l'ensemble des règles, normes, réseaux mobilisés par les agents dans le cadre de leurs échanges non marchands" (Angeon et Callois, 2004a, 1). C’est pourquoi notre analyse s'appuiera sur la notion de capital social, concept multiforme qui permet d'analyser les mécanismes économiques par lesquels les facteurs sociaux (échanges informels, structures des réseaux sociaux, pratiques solidaires) influent sur le développement territorial. La théorie économique redécouvre, finalement, l'importance de facteurs comme la confiance, la cohésion sociale ou la proximité, qui peuvent réduire les coûts de transaction. Ainsi, à côté du marché (de la concurrence) et de la hiérarchie (l'autorité), la coopération (l'association, les réseaux) s'impose comme un mode de coordination de l'activité économique (Favreau 2008, 75). Les approches du capital social et de la proximité constituent des cadres d'analyse appropriés aux questions du développement des territoires (Angeon et al., 2006). Si le capital social désigne les normes et les valeurs qui régissent l'action collective, il est aussi l'expression de la territorialité des sociétés (Loudiyi et al., 2004).

En Algérie, l'un des défis majeurs est aujourd'hui de mobiliser les populations et les instances locales pour les faire travailler ensemble (Donsimoni et Perret, 2008). Les autorités algériennes, qui souhaitent relancer l'artisanat, s'orientent

\footnotetext{
${ }^{1}$ IREGE, Université de Savoie. Cecile.Perret@univ-savoie.fr
} 
actuellement vers la création de Nuclei d'entreprises, regroupements d'entreprises qui se réunissent périodiquement autour d'un animateur afin d'identifier leurs problèmes communs et pour ensemble trouver des solutions. L'article analyse comment les Nuclei d'entreprises peuvent créer du capital social et redynamiser un territoire. La première partie présente les changements de la logique d'action des entrepreneurs et du rôle de l'État intervenus en Algérie depuis le milieu des années 1980. La deuxième examine la nature du capital social et son impact sur le développement territorial. Enfin, la troisième présente la reconfiguration des rapports État/Marché/Société civile à travers l'analyse de la mise en place des Nuclei d'entreprises sur la base de données originales collectées auprès de douze artisans de la wilaya de Tizi-Ouzou.

\section{LOGIQUE D'ACTION/CAPITAL SOCIAL ET RÔLE DE L'ÉTAT}

Au milieu des années 1980 avec le désengagement de l'État "l'entreprise privée devient " un enjeu clé et une alternative incontournable susceptible de trouver une démarche innovante en vue de valoriser les potentialités existantes tout en capitalisant les ressources humaines locales" (Nemiri-Yaici, 2006). Les années 1990 et le passage à l'économie de marché verront naître de nombreux dispositifs de soutien à la création d'entreprises et à la consolidation du secteur privé. Tous ces changements transforment "les anciennes normes économiques et, par voie de conséquence, le comportement de l'entrepreneur algérien" (Melbouci, 2006, 8).

\subsection{Le tournant des années 1990}

Pour analyser l'évolution de l'esprit entrepreneurial en Algérie à la lumière du concept de capital social, plusieurs définitions sont utiles : celle de Woolcok et Narayan (2000) (angle des normes et réseaux), celle de Coleman (1988), celle de Putnam (1995) (théorisation de l'action rationnelle centrée sur des déterminants sociaux) et enfin celle de Lin (2001) (angle des ressources). Deux approches sont à distinguer : celle dite réticulaire, qui se penche sur la nature du capital social (ressource produite et valorisée au sein d'un réseau) et celle, fonctionnaliste, qui caractérise le capital social selon ses effets.

Woolcok et Narayan (2000, in Loudiyi et al., 2004) en définissant le capital social comme l'ensemble des normes et des réseaux qui facilitent l'action collective lui confèrent deux dimensions : la première le lie aux normes et aux valeurs (règles informelles) qui régissent les interactions entre agents et la seconde le détermine par ses caractéristiques structurelles. Dès lors, le capital social désigne le cadre formel au sein duquel s'établissent les relations entre les agents économiques. Le capital social est alors constitué des institutions formelles et informelles qui facilitent l'action collective.

Putnam (1995) définit le capital social comme les réseaux et normes de réciprocité qui lui sont associées. Il a une valeur donnée pour les individus qui 
font partie du réseau. Le capital social d'un individu est donc de façon claire un ensemble de relations formelles ou informelles qui résultent d'une stratégie. L'ordre du marché et de l'organisation bureaucratique sont "balayés" au profit des rapports entre les individus basés sur la confiance et la réciprocité (Boutillier et Uzunidis, 2006, 15-16).

Enfin, Lin (in Angeon et Callois, 2004 a et b) définit le capital social comme une richesse potentielle incorporée dans la structure sociale et qui peut être (mais pas nécessairement) mobilisée en cas de besoin. Cette conception rejoint celle adoptée par Bourdieu (1980 in Angeon et al., 2006) qui définit le capital social comme "l'ensemble des ressources actuelles ou potentielles qui sont liées à la possession d'un réseau durable de relations plus ou moins institutionnalisées d'interconnaissance et d'interreconnaissance."

Pour Coleman (1988) le capital social est une ressource incorporée dans les relations interindividuelles, une forme particulière de capital qui rend possible l'action sociale; il est à l'origine des relations développées entre les agents (capacité à faire circuler l'information, existence de règles et de sanctions qui s'imposent dans une communauté particulière). Le capital social est caractérisé par ses effets : il facilite l'action entre les individus. Coleman met également en évidence l'existence de liens entre les dimensions micro et macroéconomiques, puisque les ressources (privées) qu'un individu retire de la valorisation de son capital social ont des répercussions au plan macroéconomique.

Les institutions internationales ont compris l'enjeu de ce concept multiforme. L'OCDE lui attribue une place de taille dans l'amélioration du bien-être social. La Banque mondiale, de son côté, reconnaît son importance dans la lutte contre la pauvreté car il comble le vide laissé par les institutions, piliers de la croissance économique. Il apporte un support aux relations économiques informelles qui reposent sur des liens de confiance et de solidarité réciproques (Boutillier et Uzunidis, 2006, 94).

Dans le cas de l'Algérie, Melbouci (2006) distingue deux périodes : les années 1962-1990 et les années post 1990. La première période est caractérisée par un environnement administré, stable et structuré. Durant cette période, la stratégie utilisée par l'entrepreneur algérien était axée sur l'accumulation du capital social qui, suivant la définition de Boutillier et Uzunidis (1999), se décompose en un capital-relations, un capital-connaissances et un capital-financier. En Algérie, du fait que l'entrepreneur était "mal vu et accusé, même, d'une appartenance à une classe de "bourgeoisie exploiteuse" par la charte nationale de 1976" (Melbouci, 2006, 7), l'accumulation du capital-financier n'est pas primordiale puisque l'entrepreneur ne vise pas à agrandir son affaire de peur qu'elle soit nationalisée. Par contre, le capital-relations (institutionnelles et informelles) est la base de toute stratégie des entrepreneurs algériens. Pour Melbouci (2006), ces changements ont aiguisé l'esprit entrepreneurial : les entrepreneurs sont passés d'une logique d'action patrimoniale PIC (Pérennité/Indépendance/Croissance) à une logique d'action CAP (Croissance/Autonomie/Pérennité) (Marschenay, 2004). Les PIC sont mus par une logique d'action essentiellement patrimoniale, 
tandis que les CAP le sont par une logique de valorisation du capital plutôt que d'accumulation.

Depuis 1990, la création des petites et moyennes entreprises (PME) a connu un mouvement ascendant : 60\% des PME existantes en 2000 ont été créées depuis 1994 (adoption d'un nouveau code des investissements, en octobre 1993 et entrée en vigueur du programme d'ajustement structurel, en mai 1994). Les dispositions relatives au développement des investissements sont renforcées en 2001 et une nouvelle loi d'orientation sur la PME, qui va dans le sens de la promotion de l'entrepreneuriat (Assala, 2006), voit le jour. Le secteur privé est aujourd'hui prédominant au sein de l'économie algérienne. Fin 2006, les PME privées et les activités artisanales représentaient 99,8\% du total des PME.

\subsection{Le retour de l'État dans le champ économique}

Parallèlement à ces changements, l'économie informelle algérienne prend de l'ampleur durant la décennie 1980 du fait de la spécialisation de l'offre à l'échelle internationale. En outre, en Algérie, une proportion non négligeable des entrepreneurs utilise le recours aux mécanismes traditionnels de financement familial et le poids de l'économie informelle est important dans toutes les branches d'activités.

Dans les années 1990, le marché des biens, qui est encore caractérisé par une insuffisance de l'offre, engendre inflation et commerce illicite (trabendo). Le commerce ambulant, restreint dans les années 1980, prolifère (Adair, 2002). Tandis que la demande de travail se réduit (en particulier dans les entreprises publiques qui tendent à accroître la part de leur salariat non permanent), on assiste à une extension de l'offre de travail des ménages et du chômage, ainsi qu'à une augmentation de la pluri-activité, notamment pour les actifs disposant d'un emploi stable (Ibid.). Même si la pluri-activité n'est pas véritablement appréhendée, en 1996, parmi les 2866000 salariés permanents, 23\% ont déclaré exercer une activité secondaire dont le revenu s'élevait environ à 3\% du salaire moyen (Ibid.). Depuis les années 1990, une part de la jeunesse qui se sent oubliée et/ou qui n'est pas motivée par un salaire moyen peu élevé, ou qui ne peut trouver un emploi, se tourne vers l'informel et le trabendo. À la fin des années 1990, le phénomène trabendo s'étend et touche de nombreux secteurs: les marchands de produits de piètre qualité et/ou contrefaisants de grandes marques occidentales présentent leurs produits au vu et au su de tous. Ces vendeurs ne sont pas exactement des vendeurs à la sauvette, puisque de véritables marchés informels permanents ont vu le jour un peu partout sur le territoire depuis quelques années et que les produits contrefaisants peuvent aussi se trouver dans des boutiques légales (Perret et Gharbi, 2008). Si, comme le souligne Marschenay (2004, 237-238), ce n'est que dans l'acte de création d'entreprises que l'entrepreneuriat trouve sa concrétisation la plus évidente, alors la création d'entreprises même informelles et de petite taille en est également un signe. Si les Algériens consomment presque naturellement des produits contrefaisants sans crainte de leur dangerosité (Perret et Gharbi, 2008), 
les autorités prennent conscience des dangers de l'explosion du trabendo et de "l'argent facile" qu'il procure à la jeunesse (danger, à terme, sur le capital humain de jeunes peu incités à poursuivre leurs études, manque d'attrait pour des métiers de l'artisanat traditionnel...). Le pays est aujourd'hui riche de ses ressources naturelles (augmentation des prix du pétrole) et le gouvernement profite de cette manne pour se tourner vers les petites structures, relancer l'artisanat et favoriser le développement local. Si l'Algérie est encore confrontée aujourd'hui à un processus de transition difficile, une exigence de changement de gouvernance émerge. Le problème des autorités algériennes est d'inciter à la création d'entreprises formelles, ou de tenter de formaliser une partie de l'activité informelle. Dans cette perspective, la notion de capital social, qui peut être cernée à partir de différentes variables (variable normative, variable relationnelle, variable cognitive et variable confiance) se révèle féconde. L'association de ces quatre variables est indispensable à la stabilité du système car elles interviennent à différents niveaux. La variable relationnelle fait référence au principe de réciprocité, sans lequel aucun échange (formel ou informel) n'est envisageable à long terme. La variable cognitive assure l'adaptation du mode de fonctionnement du réseau dans un environnement évolutif. La variable normative délivre aux individus les codes à respecter pour être admis dans le réseau. La variable confiance concerne la fiabilité du système social ou la certitude que même en l'absence de sanctions formelles ou informelles, des normes de réciprocité ou des comportements "vertueux" émergent, que ce soit envers les institutions ou envers les personnes proches (amis, famille...). Cette variable renferme, d'une part, la fiabilité du système et, d'autre part, la confiance diffuse envers la société et les institutions.

Sur quelles variables les autorités algériennes peuvent elles agir? Comment redonner confiance dans les institutions? Cela peut passer par des mesures d'accompagnement à la création d'entreprises (conseil, formation, accès au financement pour des projets de micro-entreprises...) et en réinvestissant le champ économique laissé de côté durant les années d'insécurité civile.

\section{NATURE DU CAPITAL SOCIAL ET DÉVELOPPEMENT TERRITORIAL}

Nous devons revenir à une appréhension plus concrète du concept de capital social (cf. Angeon et Callois, 2004a et b).

\subsection{Nature du lien social}

Collier (1998) distingue deux formes de capital social interdépendantes: le capital social gouvernemental (institutions ou organisations mises en place par l'État ou le secteur public) et le capital social civil (organisations émanant de la société civile). Uphoff (2000) différencie le capital social structurel (organisations dans lesquelles agissent les agents) et le capital social cognitif (processus mentaux des individus, valeurs, normes, croyances... qui les 
prédisposent à la coopération.). Ces deux visions permettent de dresser une typologie de la nature concrète du capital social à la manière de Sirven (2000). Le capital social cognitif peut avoir un impact sur le capital social structurel dans la mesure où les croyances en certaines valeurs (démocratiques, par exemple) poussent les agents à se regrouper de façon formelle (en associations, en organisations non gouvernementales). Les règles partagées correspondent à un ensemble "d'institutions invisibles" (capital social civil cognitif de la typologie de Sirven (2000) qui facilitent la compréhension entre les agents (Angeon et al., 2006).

La nature des liens entre les agents a été précisée par la typologie proposée par la Banque mondiale (2000) qui distingue le lien qui unit (bonding), le lien qui lie (linking) et le lien qui relie (bridging). Nous nous limiterons ici à l'analyse de la nature des liens concernant le capital social civil. Les liens de type bonding unissent des individus de statut identique (liens horizontaux) au sein d'une même communauté. S'ils caractérisent des relations de type communautaire, c'est-à-dire de personnes adhérant à un même système de représentation, ils tendent vers une "fermeture relationnelle" (Coleman, 1988), voire une forme de discrimination. Ces liens peuvent être ceux existant au sein d'une famille, d'une tribu, d'un village, d'une communauté d'appartenance... Pour appréhender l'importance de ce type de liens, des indicateurs tels que la taille des familles ou le fait d'être ou de "se sentir" kabyle, ou non, peuvent être retenus.

Les liens de type linking caractérisent des interactions entre des agents aux statuts différents. Ils sont dits verticaux. Ces liens inter-groupes nécessitent d'être réaffirmés et se caractérisent par des transactions de réciprocité qui obligent à la poursuite des échanges. Selon Angeon et al. (2006), la fréquence des interactions tend à déboucher sur la convergence des représentations.

Enfin, les liens de type bridging lient des agents distants (cette distance peut être géographique ou le lien n'est pas activé en permanence). Le bridging social capital désigne un réseau virtuel. Les relations peuvent être ponctuelles, discontinues, et les règles respectées s'apparentent à une convention sans engagement réciproque) (Ibid.). Le pourcentage d'émigrés d'un territoire donné, la part des membres de la famille qui ont émigré... constituent des indicateurs de ces liens. En cas d'activation temporaire d'un lien de type bridging, les logiques d'appartenance et/ou de similitude s'enclenchent et ne perdurent que le temps de la relation (Ibid.). C'est le cas, par exemple, lorsqu'une personne active un lien extérieur au territoire (l'émigré) pour lui demander une aide financière ponctuelle pour la réalisation d'un projet.

On peut également penser que les caractéristiques sociologiques (culture en générale) ont un impact sur le capital structurel gouvernemental (dynamisme des élus locaux, etc.). Angeon et al. remarquent dans leur étude une analogie des comportements institutionnels et de ceux des habitants : là où l'on observe un tissu associatif dense, on constate un dynamisme des élus locaux. 


\subsection{Nature des liens et impacts sur le développement}

Les coordinations locales non marchandes ont-elles une incidence sur le développement économique ? Répondre à cette question implique de distinguer les mécanismes auxquels les coordinations entre agents renvoient. Les coordinations locales génèrent deux types d'externalités positives : le premier se rattache à des questions de collecte et de circulation de l'information et le second se rapporte à l'action collective (Angeon et al., 2006). Les liens de proximité forts entre acteurs (cohésion locale ou modalités de coordination entre acteurs) sont présentés comme un élément explicatif discriminant du développement territorial. Femandez et Nichols (2002) ont également montré à partir d'une enquête menée dans la Silicon Valley (Californie) que le capital social basé sur des liens raciaux et ethniques (bonding social capital) a un effet sur le développement du capital social qui relie les différents groupes raciaux et ethniques entre eux (bridging social capital). Si les liens de type Bridging sont parfois qualifiés de faibles, leur activation a des conséquences non négligeables en matière de développement via la circulation des informations, des savoirs et des techniques permise par les liens entre agents éloignés géographiquement. Les transferts de savoirs effectués lors d'une visite ponctuelle d'un membre éloigné (géographiquement et socialement) de la famille peuvent avoir une importance et ce type de liens est non négligeable en termes de flux de financement.

\subsection{Du capital social au capital spatial}

Selon Lévy (1994), chaque individu possède un capital spatial, ressource accumulable, lui permettant de tirer un avantage de la dimension spatiale de la société. Il se divise en capital spatial de position lié à un lieu (lieu-habitat, lieuville...) et en capital spatial de situation lié à une aire (espace que l'individu s'approprie par les mobilités en maitrisant les distances). Habitat et déplacements sont des éléments importants du capital spatial (Loudiyi et al., 2004). Si le capital social est un ensemble d'interactions entre des acteurs localisés, le capital spatial est intéressant pour l'étude des inégalités sociales et spatiales. Tout territoire, même de développement spatial limité, est constitué d'objets matériels (routes, infrastructures) et animé par des réseaux sociaux (Debardieux, 2002 in Loudiyi et al., 2004). La configuration spatiale est la résultante de l'agencement des acteurs qui interagissent entre eux. Créer et renforcer le capital social pour engendrer un développement territorial suppose de repérer l'ensemble des formes du capital social qui permettent aux groupes sociaux d'un territoire de maitriser les futures évolutions (Loudiyi et al., 2004).

\section{VERS UNE RECONFIGURATION DES RAPPORTS ÉTAT/MARCHÉ/SOCIÉTÉ CIVILE ?}

L'importance du capital social en Kabylie, territoire à identité forte, porte à étudier les nouvelles approches de l'entreprise, telle que la gestion collective, 
dont l'objet concerne le regroupement de besoins, le partage de besoins ou l'échange (résidus de productions...). Concrètement, comment peut se traduire une gestion collective? Par exemple, il ne sera pas plus complexe d'effectuer une commande groupée pour plusieurs PME que pour une seule.

\subsection{Nuclei et territorialisation du capital social}

Les autorités algériennes qui souhaitent relancer l'artisanat s'orientent actuellement vers la création de Nuclei. Les Chambres de l'Artisanat et des Métiers $(\mathrm{CAM})^{2}$ ou les Chambres de Commerce et d'Industrie (CCI ${ }^{3}$ participantes au programme Nuclei ont signé préalablement une convention de partenariat proposée par GTZ4-AAPOP5 (coopération algero-allemande) Qu'est-ce qu'un Nucleus ? C'est un regroupement d'entreprises d'un même secteur économique qui se réunissent périodiquement autour d'un conseiller/animateur de Nucleus pour identifier leurs problèmes communs et pour ensemble trouver des solutions (achats groupés, marketing commun, échanges d'informations sur les clients, sur les techniques...). Le conseiller/animateur se situe au cœur du dispositif. C'est l'AAPOP qui le forme et l'accompagne dans ses activités au profit des membres du (ou des) Nucleus(i). Tout Nucleus est donc adossé à une Chambre consulaire. Depuis le démarrage de cette expérience en Algérie, en juillet 2007, et avec l'appui de GTZ, plus d'un millier d'entrepreneurs se sont organisés en plus de 120 Nuclei dans les CAM pilotes. La CAM assure la gestion des cartes d'artisans, forme, organise des foires, assure des services de commercialisation et de conseils individuels, effectue des achats groupés de matières premières, dispose d'un fonds de promotion rural et de conventions avec des organismes d'assurances et, enfin, organise les élections des représentants des artisans.

Deux catégories de Nuclei sont identifiés : les Nuclei verticaux (de secteur) et les Nuclei horizontaux (les participants sont du même secteur et offrent des produits et des services identiques). Il est admis que lorsque des entrepreneurs, ou des cadres d'entreprise, ont une problématique commune, il est possible de créer un Nucleus. L'essentiel est que les individus concernés puissent s'apporter quelque chose. Selon le Directeur de la CAM de Béjaïa, le réseau Nucleus se constitue à partir de sept à huit entrepreneurs et artisans d'un même secteur ayant des préoccupations communes et proches géographiquement. D’autres entrepreneurs peuvent ensuite y adhérer, jusqu'à atteindre une vingtaine, nombre jugé maximum pour que le conseiller puisse organiser des réunions

2 Les CAM d'Alger, Bejaia, Blida, Jijel, Mostaganem, Oran, Sétif, Tipaza, Tizi Ouzou et Tlemcen sont concernées.

${ }^{3}$ Les CCI de Dahra, Seybousse et Tafna sont concernées.

${ }^{4}$ La Deutsche Gesellschaft für Technische Zusammenarbeit (GTZ) est l'Agence de coopération technique allemande pour le développement.

5 Appui aux associations professionnelles, chambres et organisations patronales. 
fructueuses. Cette approche Nucleus développée dans le monde depuis $1991^{6}$ vise, d'une part, à mobiliser les entreprises individuelles, particulièrement les PME, et, d'autre part, à initier des processus de développement structurel au sein des chambres et associations de commerce. En 2006, la wilaya de Bejaia gérait 9 Nuclei qui représentaient 123 entrepreneurs ${ }^{7}$.

Selon Loudiyi et al. (2004), trois catégories simplifiées d'acteurs existent: la première $(G)$ rassemble des individus ayant une action collective intentionnelle ou non; la deuxième (GP) est un groupe dit productif, un collectif d'acteurs ayant un but commun finalisé qui se dotent de règles communes; la troisième (I) est représentée par les acteurs institutionnels. À ces trois types d'acteurs, les auteurs associent des qualités reconnaissables (la forme prédominante) de capital social. Au groupe $G$, ils associent la recherche du "vivre ensemble" (bonding); au groupe GP ils associent la recherche du "produire ensemble" (linking) et aux acteurs institutionnels un rôle d'organisation et d'encadrement, "organiser ensemble" (bridging). Le passage d'un type d'acteur à un autre correspond à un changement de rôle et à la présence d'un lien prédominant.

\subsection{Des expériences de gestion collectives créatrices de capital social en Algérie}

En s'organisant en Nucleus, les entrepreneurs deviennent un groupe productif et sont partie prenante d'un acteur institutionnel, la CAM. Ils acquièrent une légitimité différente du fait de l'élargissement de leurs liens avec des acteurs reconnus à une échelle supérieure (ministère des PME et de l'Artisanat). Les acteurs peuvent jouer plusieurs rôles dans la réalité. Le Nucleus est lui-même générateur de capital social entre les PME du fait des échanges possibles entre tous les participants. Un Nucleus horizontal constitue un réseau localisé, c'est un système de relations à partir d'une activité commune spécialisée sur un territoire (linking territorial). Plusieurs Nuclei pouvant dépendre de la même CAM et des mêmes animateurs, cela crée du capital social de type bonding. Le programme Nucleus relie également des acteurs institutionnels de statuts différents (le Nucleus et la CAM, par exemple) : il crée du capital social de type bridging. Il y a mise en place de nouveaux réseaux d'acteurs. Le directeur ${ }^{8}$ de la CAM de Bejaia-Bouira rencontre ses trois animateurs de Nuclei chaque semaine et participe personnellement à des réunions de Nucleus.

La figure de type bridging articule des acteurs de plusieurs statuts et échelles différentes. Certains acteurs ont des statuts institutionnels situés à des niveaux d'organisation englobant (ministère, par exemple). Les objets spatiaux qu'ils créent sont de deux types: de nouvelles organisations d'acteurs (les Nuclei qui engendrent de la coopération) et de nouveaux aménagements (un pôle

\footnotetext{
${ }^{6}$ L'approche Nucleus a été initiée dans le cadre du projet de partenariat entre la Chambre des Métiers et les petites industries de Munich et plusieurs autres Chambres de Commerce et d'Industrie brésiliennes de l'État Fédéral de Santa Catarina.

${ }^{7} \mathrm{http}: / / \mathrm{www}$.Nucleus-algerie.com.

${ }^{8}$ Enquête personnelle en mars 2009.
} 
d'économie du patrimoine, par exemple). Il arrive, en outre, que les entrepreneurs des Nuclei rencontrent les pouvoirs publics (via la participation à des rencontres des responsables de l'administration centrale et des directions de wilaya), des institutions d'appui, des instituts de formation, dans un objectif de développement de leur capital social.

\subsection{Les résultats}

Que peut-on, à ce jour, retirer de cette expérience Nuclei ? Pour avoir une idée de sa réussite, ou de son échec, il faudrait étudier sur le long terme l'évolution du nombre et de la nature des entreprises créées, ainsi que de leurs liens depuis la mise en place de ce programme. Afin d'analyser de façon plus approfondie et systématique les apports de cette approche, notamment en terme de création de capital social, nous avons, grâce à l'appui du Directeur de la CAM de TiziOuzou, administré un questionnaire à des participants aux Nuclei que gère cette Chambre. Cinq bijoutiers et sept potiers ont été questionnés.

Tableau 1 : Apports de la participation à l'approche Nucleus

\begin{tabular}{|l|c|c|}
\hline & Wilaya de Tizi-Ouzou \\
\hline Aides et conseils & 5 bijoutiers & 7 potiers \\
\hline Conseils lors de la création de l'entreprise & 1 & \\
\hline Conseils dans la gestion de l'entreprise & 3 & 3 \\
\hline Partage de connaissances techniques avec les membres du Nucleus & 2 & 3 \\
\hline Conseils financiers & 3 & 1 \\
\hline Aides dans la recherche de fournisseurs plus avantageux & & 1 \\
\hline Aide dans la recherche de clients ou nouveaux clients & 1 & \\
\hline Aide à la distribution des produits (participation à des foires, des salons) & 2 & 3 \\
\hline Aide à la publicité de vos produits & 2 & 3 \\
\hline
\end{tabular}

Source : Enquête personnelle, mars 2009.

Tous ces artisans reconnaissent avoir fait de nouvelles connaissances grâce à leur participation au programme. Nous avons synthétisé dans le tableau 1 le nombre de citations à la question suivante : quels sont les conseils ou aides dont vous avez bénéficié grâce à votre participation au programme Nucleus?

\section{CONCLUSION}

S’il est trop tôt pour conclure sur la réussite de ce programme en termes de dynamisme des territoires, son impact dans la formation de capital social paraît indéniable. Une rencontre des Chambres de l'Artisanat et des Métiers de Bejaïa, Sétif et Jijel s'est tenue récemment à Bejaïa autour de ce thème "L'apport de l'approche Nucleus dans la formation du capital social des entrepreneurs et artisans"9. Notre étude, sans prétendre à l'exhaustivitée ${ }^{10}$, montre que cette

\footnotetext{
${ }^{9}$ Cette rencontre était appuyée par la coopération algéro-allemande (notamment la composante qui appuie les structures associatives professionnelles - CAM/CCI associations professionnelles) regroupant les PME et micro-entreprises (artisanat).

${ }^{10}$ Une seconde analyse, plus étendue, sera menée à la suite de cette enquête.
} 
approche Nucleus est créatrice de lien social et améliore la circulation de l'information (transmission de connaissances, par exemple). Par ailleurs, et cela est sans doute fondamental dans le cas de l'Algérie, elle permet aux pouvoirs publics de se "réapproprier" un terrain économique largement abandonné au bénéfice du terrain sécuritaire depuis la décennie noire.

Si les caractéristiques sociologiques (culture en général) ont un impact sur le capital structurel gouvernemental (dynamisme des élus locaux, par exemple) et s'il existe une analogie possible des comportements institutionnels avec celui des habitants (Angeon et al. (2006), alors une étude similaire à celle que nous avons conduite sur les Nuclei de la wilaya de Tizi-Ouzou, mais étendue à toute l'Algérie devrait pouvoir nous apporter des informations complémentaires.

\section{BIBLIOGRAPHIE}

ADAIR P. (2002) L'emploi informel en Algérie : évolution et segmentation du marché du travail, GRATICE, Université Paris XII, miméo.

ANGEON V., CALLOIS J.-M. (2004a) De l'importance des facteurs sociaux dans le développement, Premières journées du développement du GRES, Le concept de développement en débat, Université Montesquieu-Bordeaux IV, 16-17 septembre.

ANGEON V., CALLOIS J.-M. (2004b) Fondements théoriques du développement local : quels apports de la théorie du capital social et de l'économie des proximités?, Quatrièmes journées de la proximité, Marseille.

ANGEON V., CARON P., LARDON S. (2006) Des liens sociaux à la construction d'un développement territorial durable: quel rôle de la proximité dans ce processus ?, Développement durable et territoire, Dossier 7 Proximité et environnement (http://developpementdurable.revues.org/).

ASSALA K. (2006) PME en Algérie : de la création à la mondialisation, 8 ème Congrès international francophone en entreprenariat et PME, 25-27 octobre, Haute école de gestion (HEG), Fribourg, Suisse.

BANQUE MONDIALE (2000) Rapport sur le développement dans le monde 2000/2001. Combattre la pauvreté, Paris, Editions Eska, 382 p.

BOUTILLIER S., UZUNIDIS D. (2006) Travailler an XXIème siècle. Nowveaux modes d'organisation du travail, Economie, Société, Région, collection de l'Institut wallon de l'évaluation, de la prospective et de la statistique (IWEPS), De Boeck, 324 p.

BOUTILLIER S., UZUNIDIS D. (1999) La légende de l'entrepreneur : le capital social on comment vient l'esprit d'entreprise, Paris, Ed. Syros, $151 \mathrm{p}$.

COLEMAN J. S. (1988) Social Capital in the Creation of Human Capital, American Journal of Sociology, vol. 94, 95-120.

COLLIER P. (1998) Social capital and poverty, Social Capital Initiative WP $n^{\circ} 4$, The World Bank, Washingtion DC, USA.

DENIEUIL P.-N. (1999) Introduction aux théories et à la pratique du développement local et territorial. Analyse et synthèse bibliographique en écho au séminaire de Tanger (25-27 novembre), Programme focal de promotion de l'emploi par le développement des petites entreprises, Département de la création d'emplois et de l'entreprise, SEED Document de Travail nº 70, BIT-Genève, 58 p. 
DONSIMONI M., PERRET C. (2008) Capital social et développement territorial. Le cas de deux ensembles de Wilayate de Kabylie, colloque international, Développement local et gouvernance des territoires, Université de Jijel (Algérie), 3-5 novembre.

FAVREAU L. (2008) Entreprises collectives. Les enjeux sociopolitiques et territoriaux de la coopération et de l'économie sociale, Presses de l'Université du Québec, 332 p.

FEMANDEZ M., NICHOLS L. (2002) Bridging and bonding capital: Pluralist ethnic relations in Silicon Valley, International journal of sociology and social policy, vol. 22, $\mathrm{n}^{\circ} 9$ $10,104-122$.

LEVY J. (1994) L'espace légitime. Sur la dimension géographique de la fonction politique, Paris, Presses de la Fondation nationale des sciences politiques, $442 \mathrm{p}$.

LIN N. (2001) Social capital. A theory of social structure and action, Cambridge University Press, coll. "Structural Analysis in the social science", 278 p.

LOUDIYI S., ANGEON V., LARDON S. (2004) Capital social et développement territorial. Quel impact spatial des relations sociales ?, mimeo.

MARCHESNAY M., (2004) Management stratégique, Editions de l'ADREG, 284 p.

MELBOUCI L. (2006) De l'économie administrée à l'économie de marché : quelles stratégies pour l'entrepreneur algérien face à la concurrence mondiale ?, 8ème Congrès international francophone en entreprenariat et PME, 25-27 octobre, Haute école de gestion (HEG), Fribourg, Suisse.

NEMIRI-YAICI N. (2006) Profil des entreprises privées de la wilaya de Béjaïa : caractéristiques, financement et rentabilité, colloque international Création d'entreprises et territoires, Tamanrasset, 3-4 décembre, mimeo.

PERRET C., GHARBI N. (2008) La contrefaçon en Algérie. Risque et prise en compte par les entreprises. Une application aux produits cosmétiques, Cabiers du CREAD, n'85-86, Alger.

PUTNAM R. D. (1995) Bowling Alone: America's Declining Social Capital, The Journal of Democracy, 6:1, 65-78.

SIRVEN N. (2000) Capital social et développement : quelques éléments d'analyse, Centre d'économie du développement, Document de travail n57, Bordeaux, $26 \mathrm{p}$.

UPHOFF N. (2000) Understanding Social Capital: Learning from the Analysis and Experience of Participation, in Dasgupta P., Serageldin I. (ed.), Social Capital: A Multifaceted Perspective, Washington, D.C. The World Bank, 215-252.

WOOLCOK M., NARAYAN D. (2000) Social capitals: implication for development theory, research and policy, The World Bank Research Observer, vol. 15, n² 2, 225-249. 